\title{
Estudio LIFAC: evaluación de la utilidad de un libro de incidencias en farmacia comunitaria
}

\section{Pedro Gutiérrez Ríos', Fernando Abellán-García Sánchez², María José Faus Dáder', Miguel Ángel Gastelurrutia Garralda ${ }^{1,3}$, Fernando Martínez Martínez ${ }^{3}$, María Jesús Rodríguez Martínez ${ }^{1}$}

1. Farmacéutico comunitario, SEFAC Sociedad Española de Farmacia Familiar y Comunitaria, Madrid. 2. Abogado especialista en Derecho Sanitario, Coordinador de la Comisión de Bioética de SEFAC Sociedad Española de Farmacia Familiar y Comunitaria, Madrid. 3. Grupo de Investigación en Atención Farmacéutica de la Universidad de Granada.

\section{PALABRAS CLAVE}

Incidencia, libro de incidencias, dispensación excepcional, responsabilidad profesional, farmacéutico comunitario

\section{ABREVIATURAS}

ATC: clasificación anatómica, terapéutica y química.

EPA: estudio postautorización. LIFAC: libro de incidencias en la farmacia comunitaria SEFAC: Sociedad Española de Farmacia Familiar y Comunitaria.

\section{KEYWORDS}

Incidence, book of incidences. exceptional dispensing, professional responsibility, commuity pharmacist

\section{RESUMEN}

Objetivo: El farmacéutico comunitario se ve obligado en numerosas ocasiones a atender las necesidades que los pacientes le plantean en demanda de soluciones, mediante actuaciones que la estricta observación de la legalidad vigente le impide realizar pero que su preparación profesional y su cercanía al paciente, avalan. El registro escrito de este tipo de situaciones y la decisión adoptada por el farmacéutico es el objeto de este estudio, con el fin de evaluar su utilidad, categorizar e inventariar las incidencias que se presentan, servir de respaldo de su actuación y mejorar la calidad asistencial al paciente.

Métodos: Diseño multicéntrico, nacional y prospectivo. Se ofreció la participación a socios de SEFAC que registraron en formato electrónico (Libro de Incidencias) las incidencias producidas en el quehacer profesional diario según una plantilla de tipos predefinidos acordada por la Comisión de Bioética de SEFAC.

Resultados: 170 farmacéuticos comunitarios registraron 1.565 incidencias. 64,7\% dispensaciones excepcionales resueltas en un 29,7\% mediante la ficha de paciente y un 14,5\% tras entrevista farmacéutico-paciente. El 35,3\% correspondieron a otros tipos de incidencias: no dispensación por razones clínicas $(21,5 \%)$ y prescripción incorrecta $(14,5 \%)$. El grupo terapéutico más implicado fue el de los antiinfecciosos $(20,1 \%)$ y relacionados con el SNC $(18,7 \%)$.

Conclusiones: El estudio LIFAC pone de manifiesto que el farmacéutico comunitario resuelve situaciones en las que de una u otra manera la salud de los pacientes se ve comprometida. Los participantes perciben que el registro de las incidencias es de gran ayuda para la justificación de su actuación profesional en situaciones complejas o comprometidas.

LIFAC study: assessment of the usefulness of an incident book in community pharmacy

\section{ABSTRACT}

Aim: The community pharmacist is often obliged to deal with patient needs put to them in terms of demanding solutions, by means of actions that strict observation of prevailing legality prevents carrying out but which comes guaranteed by their professional training and proximity to the patient. Strict record of this kind of situation and the decision taken by the pharmacist is the object of this study, with the purpose of assessing its usefulness, categorizing and making an inventory of the incidents that present, serving as a support for their action and improving the quality of patient care.

Methods: Multicenter, national and prospective design. SEFAC (Spanish Society for Community Pharmacy) members were given the opportunity to take part; they made an electronic record (Incident $B o o k$ ) of the incidences that occurred in daily professional work according to a preset template agreed by the SEFAC Bioethics Committee.

Results: A total of 170 community pharmacists recorded 1565 incidences; $64.7 \%$ cases of exceptional dispensing were resolved in $29.7 \%$ and $14.5 \%$ by means of the patient record and after a pharmacist-patient meeting, respectively. A total of 35.3\% corresponded to other kinds of incident: no dispensing for clinical reasons (21.5\%) and incorrect prescription (14.5\%). The therapeutic group most involved was anti-infectious drugs (20.1\%) and related to the CNS (18.7\%).

Conclusions: The LIFAC study highlights that the community pharmacist resolves situations in which patient health is in some way seen as compromised. Participants perceive that the record of incidents is of major help to justify their professional work in complex or compromised situations.

Financiación: El estudio LIFAC contó con la colaboración económica de la Fundación Astra Zéneca. Conflicto de intereses: Los autores declaran no existir conflicto de intereses en relación con el contenido del artículo. Cite este artículo como: Gutiérrez P, Abellán-Garcia F, Faus MJ, Gastelurrutia MA, Martínez F, Rodríguez MJ. Estudio LIFAC: evaluación de la utilidad de un libro de incidencias en farmacia comunitaria. Farmacéuticos Comunitarios. 2015 Sep 01; 7(3):14-18. doi:10.5672/FC.2173-9218. (2015/Nol7).003.03

Autor para correspondencia: Pedro Gutiérrez Ríos (pgutierrez@cnoo.es).

ISSN 1885-8619 @SEFAC (Sociedad Española de Farmacia Familiar y Comunitaria). Todos los derechos reservados. 


\section{Introducción}

En las conclusiones de los trabajos de la Comisión de Bioética de la Sociedad Española de Farmacia Familiar y Comunitaria (SEFAC) desprendidas del manual Recetas sencillas para preguntas complejas. Cómo resolver los 15 conflictos tipo en la relación asistencial farmacéutico-paciente, editado en 2009 (1), se puso de manifiesto la conveniencia de la llevanza en la farmacia comunitaria de un Libro de Incidencias que permitiera recoger $\mathrm{y}$ dejar constancia escrita de las motivaciones que subyacen en las decisiones clínicas más complejas o comprometidas que se ve obligado a adoptar en ocasiones el farmacéutico comunitario en el desarrollo de las funciones que le son propias.

De hecho, el objetivo de una mejor asistencia al paciente que justifica de manera fundamental la tramitación de una historia clínica en cualquier centro asistencial, es extrapolable, con las debidas adaptaciones, a la actividad del farmacéutico comunitario en la prestación de servicios de atención farmacéutica.

La generación de una documentación clínica adecuada por parte de cualquier profesional sanitario debe entenderse como un factor de calidad asistencial en la medida que permite dejar rastro de la actividad que desarrolla y de las razones que sustentan determinadas decisiones. También faculta para hacer un mejor seguimiento de los pacientes y usuarios favoreciendo así una práctica profesional más segura desde el punto de vista de la responsabilidad legal.

Todo lo anterior constituye además ámbitos de futuro para la profesión, que debe ir profundizando en el terreno de los servicios profesionales. Cara al futuro hay que ahondar en la implantación de nuevos servicios profesionales en la atención al paciente, tanto en lo que se refiere al medicamento en sí como a su bienestar, mantenimiento de la salud en general y prevención de enfermedades. No obstante, para llevar a cabo este tipo de servicios en la farmacia comunitaria, previamente han de demostrar su eficiencia y valor añadido por medio de estudios que lo avalen.

En este punto hay que significar la existencia de determinadas ineficiencias en el sistema sanitario, muy especialmente en lo que entendemos como "dispensación excepcional”, es decir, aquellos supuestos en los que, en ausencia de la receta física, el farmacéutico comunitario se considera en la obligación de dispensar el medicamento que se le requiere con el fin de evitar perjuicios en la salud del paciente (considérese enfermos crónicos, agudización o prolongación de procesos patológicos, etc.). En todos estos casos se contempla la dispensación de medicamentos que han sido prescritos por el médico y que una situación de urgencia o necesidad justifique la dispensación por parte del farmacéutico y bajo su criterio profesional (2).

Precisamente de cara a estos casos de dispensación excepcional, aunque no solamente en ellos, el $\mathrm{Li}$ bro de Incidencias puede resultar un instrumento válido para la justificación clínica de las decisiones profesionales. $\mathrm{Al}$ análisis de la relevancia y utilidad de este libro para la farmacia comunitaria (concebido en formato electrónico) y a la definición de su propio diseño responden los objetivos básicos de este estudio.

La Comisión de Bioética de SEFAC, a efectos del estudio LIFAC, define incidencia como: "Cualquier acontecimiento anómalo que se produzca en la asistencia de usuarios o pacientes en la farmacia comunitaria que conduzca al farmacéutico a la toma de una decisión compleja o comprometida de la que pudiera derivarse alguna responsabilidad clínica, profesional, deontológica o legal” (3).

\section{Objetivos}

El objetivo principal del estudio es evaluar la utilidad de la llevanza en la farmacia comunitaria del Libro de Incidencias propuesto por la Comisión de Bioética de SEFAC, como factor de mejora de la calidad asistencial a los pacientes.

Asimismo este estudio tiene una serie de objetivos específicos que son los siguientes:

1. Diseñar un modelo de Libro de Incidencias sobre la base de la hoja de registro de incidencias y de las instrucciones para su utilización propuestas por la Comisión de Bioética de SEFAC.

2. Evaluar la llevanza del citado libro como instrumento preventivo en materia de responsabilidad profesional, en la medida que permita al farmacéutico explicar y probar $a$ posteriori las circunstancias clínicas en las que tuvo que tomar decisiones comprometidas o complejas.

3. Inventariar y categorizar los principales tipos de incidencias en materia de responsabilidad profesional que se producen en las tareas asistenciales del farmacéutico comunitario.

4. Proponer medidas de difusión del Libro de Incidencias para todo el colectivo de la farmacia comunitaria española, a través de acuerdos con otras instituciones y corporaciones públicas, en caso de que los resultados obtenidos para los anteriores objetivos así lo aconsejen.

\section{Material y métodos}

Se trata de un estudio con diseño multicéntrico, nacional y prospectivo, no postautorización (No-EPA), dirigido a la implantación del Libro de Incidencias propuesto por SEFAC.

La participación en el estudio se ofreció a socios de SEFAC. Se habilitó un único Libro de Incidencias por farmacia si bien podían colaborar todos los farmacéuticos que prestaran sus servicios en la farmacia, siempre bajo la coordinación del farmacéutico participante.

El logro del objetivo principal se valora de acuerdo con el grado de éxito que se consiga respecto a los objetivos específicos y a la satisfacción general de los participantes, constatada en el cuestionario diseñado al efecto.

Para la consecución del objetivo específico 1 (diseño del Libro de Incidencias) se parte de un modelo de hoja en formato electrónico elaborado por la Comisión de Bioética de SEFAC, que tras su cumplimentación se remite telemáticamente al gestor de datos del estudio.

Para la consecución del objetivo específico 2 (prevención en materia de responsabilidad profesional) se pone en contraste la tipología de las incidencias registradas con los casos en esta materia que obran en la base de datos del servicio SEFAC Bioéticolegal que presta la firma Derecho Sanitario Asesores, que constituye un importante referente en este terreno.

Por lo que respecta al objetivo específico 3 (categorización de los diferentes tipos de incidencia) se parte de una plantilla de tipos predefinidos creada por la Comisión de Bioética de 
Tabla 1. Tipos de incidencias registradas

\begin{tabular}{|c|c|c|}
\hline \multicolumn{2}{|l|}{ Tipo de incidencia } & n (\%) \\
\hline \multirow{11}{*}{$\begin{array}{l}\text { Dispensaciones excepcionales } \\
1.012(64,7 \%)\end{array}$} & Ficha de paciente en la farmacia & $279(17,8)$ \\
\hline & Informe clinico & $219(14,1)$ \\
\hline & Entrevista farmacéutico-paciente & $136(8,7)$ \\
\hline & Copia de la receta & $127(8,1)$ \\
\hline & Prescripción no física & $81(5,2)$ \\
\hline & Discrepancia de juicio entre farmacéutico y prescripción médica & $73(4,7)$ \\
\hline & Receta electrónica & $32(2,0)$ \\
\hline & Otras pruebas de uso & $22(1,4)$ \\
\hline & Prescripción con defectos administrativos & $21(1,3)$ \\
\hline & Otra documentación & $14(0,9)$ \\
\hline & Fax & $8(0,5)$ \\
\hline \multirow{11}{*}{$\begin{array}{l}\text { Otras incidencias } \\
553(35,3 \%)\end{array}$} & Razones clínicas & $134(8,6)$ \\
\hline & No dispensación por falta de prescripción correcta & $94(6,0)$ \\
\hline & Queja de paciente & $81(5,2)$ \\
\hline & Sustitución de medicamentos & $71(4,5)$ \\
\hline & Paciente no acepta prescripción médica por desacuerdo & $66(4,2)$ \\
\hline & No dispensación por motivos de conciencia & $43(2,7)$ \\
\hline & Paciente no acepta prescripción médica por motivos económicos & $25(1,6)$ \\
\hline & No dispensación por falta de medicamento & $20(1,3)$ \\
\hline & Problemas con SPD & $13(0,8)$ \\
\hline & Discrepancia entre titular y adjunto & $4(0,3)$ \\
\hline & Dispensación a menores & $2(0,1)$ \\
\hline & & $1.565(100,0)$ \\
\hline
\end{tabular}

SEFAC y en cuanto al objetivo específico 4 (propuesta de difusión del Libro de Incidencias) se evalúa la frecuencia de registro de las incidencias.

Previo al inicio de la fase de estudio se habilitó una plataforma informática para la formación voluntaria en materia de bioética, normativa farmacéutica y sanitaria, y en la que se incluyeron casos prácticos tipo para ilustrar el manejo de la hoja de registro y la resolución de incidencias.

\section{Resultados/Discusión}

El registro de incidencias ha estado abierto en el periodo comprendido entre el 15 de junio de 2011 y el 15 de mayo de 2012. Se inscribieron un total de 170 farmacéuticos comunitarios participantes que llevaron a cabo el registro de 1.565 incidencias (9,2) farmacéutico).

El 64,7\% de las incidencias registradas corresponden a dispensaciones excepcionales mientras que el 35,3\% restante corresponde a otros tipos de incidencias (tabla 1). El 74,0\% del total de incidencias se han producido con el propio paciente mientras que el otro $26,0 \%$ con el cuidador o representante.

En lo que se refiere a dispensación excepcional, el justificante de la existencia de prescripción viene dado por algún documento diferente a la propia receta o alguna otra prueba fehaciente de uso del medicamento. Cabe destacar que en el 44,2\% de los casos de dispensación excepcional la dispensación se llevó a cabo a la vista de la ficha de paciente obrante en la propia farmacia $(29,7 \%)$ o bien tras la entrevista farmacéutico-paciente $(14,5 \%)$ inherente al servicio de dispensación en el contexto de la atención farmacéutica. La inmensa mayoría de los casos fueron para resolver situaciones de urgencia o necesidad $(86,7 \%)$ en las que las circunstancias dan cobertura ética y legal a la actuación del profesional, entendiéndose como adelantamiento de la medicación ya prescrita pero sin que medie a tiempo real la receta física o posibilidad de comprobación electrónica.

El 35,3\% de los registros, que corresponde a otros tipos de incidencias (no relacionadas con dispensación excepcional), aunque se distribuye bastante homogéneamente entre la mayor parte de ellas, cabe destacar 


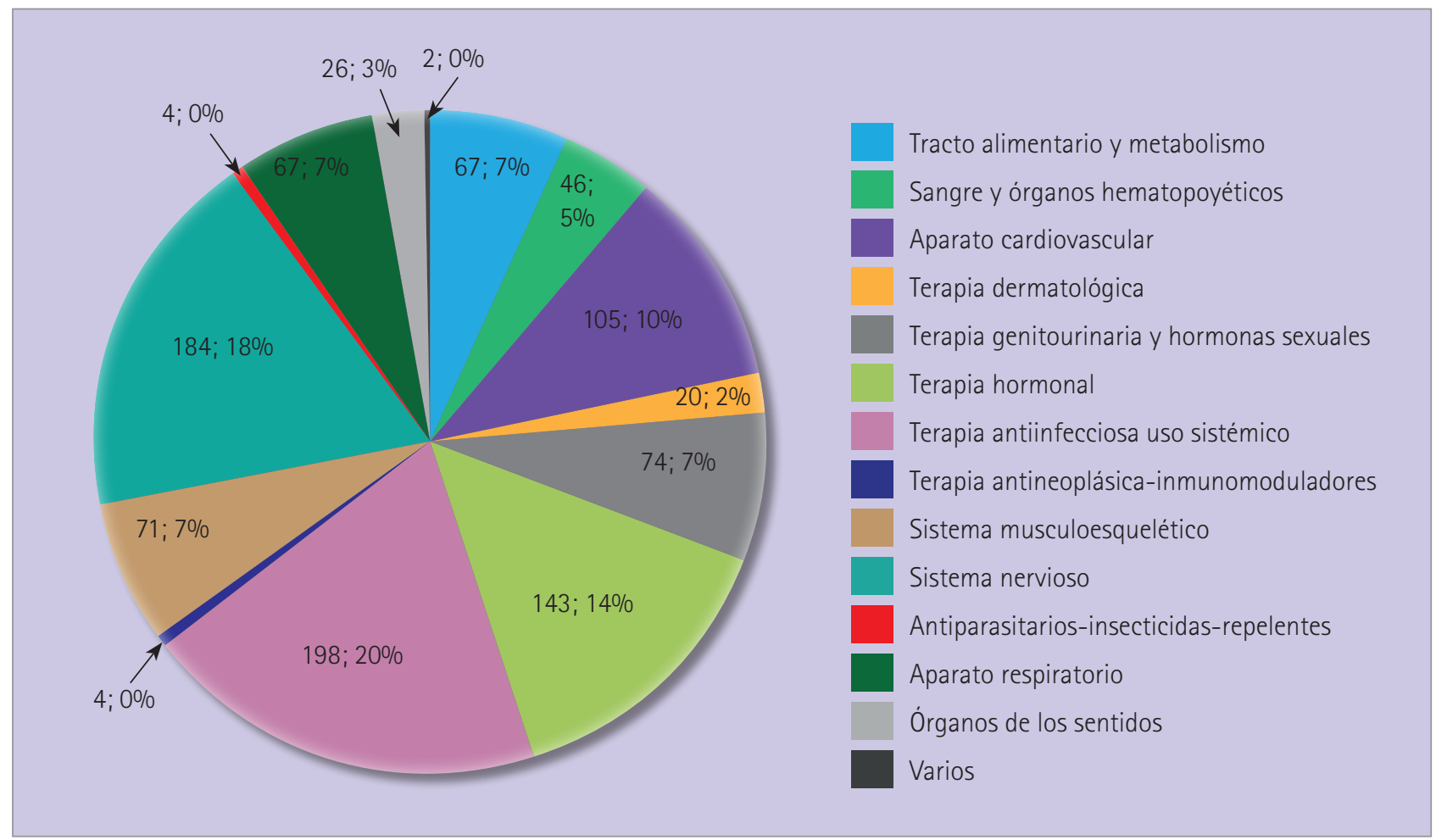

Figura 1. Grupos de medicamentos implicados en las incidencias registradas

las relacionadas con "razones clínicas" $(21,5 \%)$, "falta de prescripción correcta” (14,5\%), y mención especial a la "no aceptación de la prescripción médica por parte del paciente" debido a "desacuerdo con el médico" (10,5\%) y "por motivos económicos" (4,0\%), causa que en la actualidad se está incrementando debido a la situación económica que nuestro país sufre, aumento del copago de los medicamentos y desfinanciación de un buen número de ellos por el Sistema Nacional de Salud.

En cuanto a medicamentos implicados los ha habido de todos los grupos terapéuticos según la clasificación Anatómica, Terapéutica y Química (ATC), destacando los antiinfecciosos $(20,1 \%)$ y los relacionados con el sistema nervioso $(18,7 \%)$ (figura 1).

En lo que se refiere a la resolución de las incidencias, en el 57,0\% de los casos se dispensó el medicamento solicitado, en un $7,1 \%$ un medicamento diferente al solicitado y en el $27,4 \%$ no se dispensó ningún medicamento. Se derivó al médico o al centro de urgencias en el $28,8 \%$ de las ocasiones.

La justificación aducida por el farmacéutico para la resolución de la incidencia fue en la mayoría de los casos en función del paciente al considerar que se adoptaba la mejor solución para satisfacer sus necesidades terapéuticas o a la vista del estado del paciente $(52,1 \%)$. A su vez se registraron 90 comentarios a estas justificaciones, poniéndose de manifiesto en 60 de ellas que el farmacéutico soluciona problemas de orden sanitario y en las 30 restantes de índole administrativo.

La valoración del estudio LIFAC por parte de los farmacéuticos participantes (figura 2) ha sido muy positiva sobre todo en los aspectos de "Ayuda a reunir casuística para mejorar la percepción profesional del colectivo farmacéutico" (8,4 sobre 10$)$, de "Le ayuda a justificar su actuación profesional en situaciones comprometidas" $(8,3)$ y "Le ayuda a aumentar su responsabilidad en la dispensación" $(7,7)$. La pregunta de si “¿Cree que el Libro de Incidencias debería ser de tenencia obligatoria en la farmacia comunitaria?" obtiene una calificación de 6,6 puntos. Se señala el dato de que el $85 \%$ de los participantes han registrado al menos una incidencia por semana.

El estudio LIFAC pone de manifiesto, a la vista de los resultados, que el farmacéutico comunitario resuelve de hecho situaciones que se dan con frecuencia en el ejercicio diario de la profesión, en las que de una u otra manera la salud de los pacientes se ve comprometida, y que no puede eludir sin hacer dejación de su responsabilidad como agente sanitario en la primera línea de la atención primaria de salud. Su actuación profesional, asumiendo una responsabilidad apoyada en su preparación científica, en aras a un bien mayor consistente en la mejora de la salud de los usuarios y pacientes, a los que se debe, supone al mismo tiempo una descarga importante de la presión asistencial sobre otras estructuras de la sanidad pública, con el consiguiente beneficio sanitario y social.

Por otra parte, el sistema planificado de ordenación farmacéutica garantiza la cercanía de los establecimientos farmacéuticos comunitarios prácticamente al 100\% de la población. Este importante activo de nuestro sistema sanitario pone al farmacéutico comunitario en situación idónea para dar un servicio a la población que sería muchísimo más efectivo si se le otorgase mayor capacidad legal o, como es el caso que nos ocupa, medios que justifiquen y avalen la actuación profesional que estén reconocidos por las autoridades competentes. 
1. ¿Considera engorrosa la cumplimentación del libro de incidencias?

2. ¿Le ayuda a mejorar la calidad de la atención farmacéutica?

3. ¿Le ayuda a detectar problemas relacionados con los medicamentos?

4. ¿Mejora la relación con los pacientes?

5. ¿Le ayuda a justificar su actuación profesional en situaciones comprometidas?

6. ¿Le ayuda a aumentar su responsabilidad en la dispensación?

7. ¿Ha motivado el envío de alguna tarjeta amarilla de farmacovigilancia?

8. ¿Ayuda a reunir casuistica para mejorar la percepción profesional del colectivo farmacéutico?

9. ¿Mejora la relación profesional con los farmacéuticos de la farmacia donde trabaja?

10. ¿Este libro debería ser de tenencia obligatoria en la farmacia comunitaria?

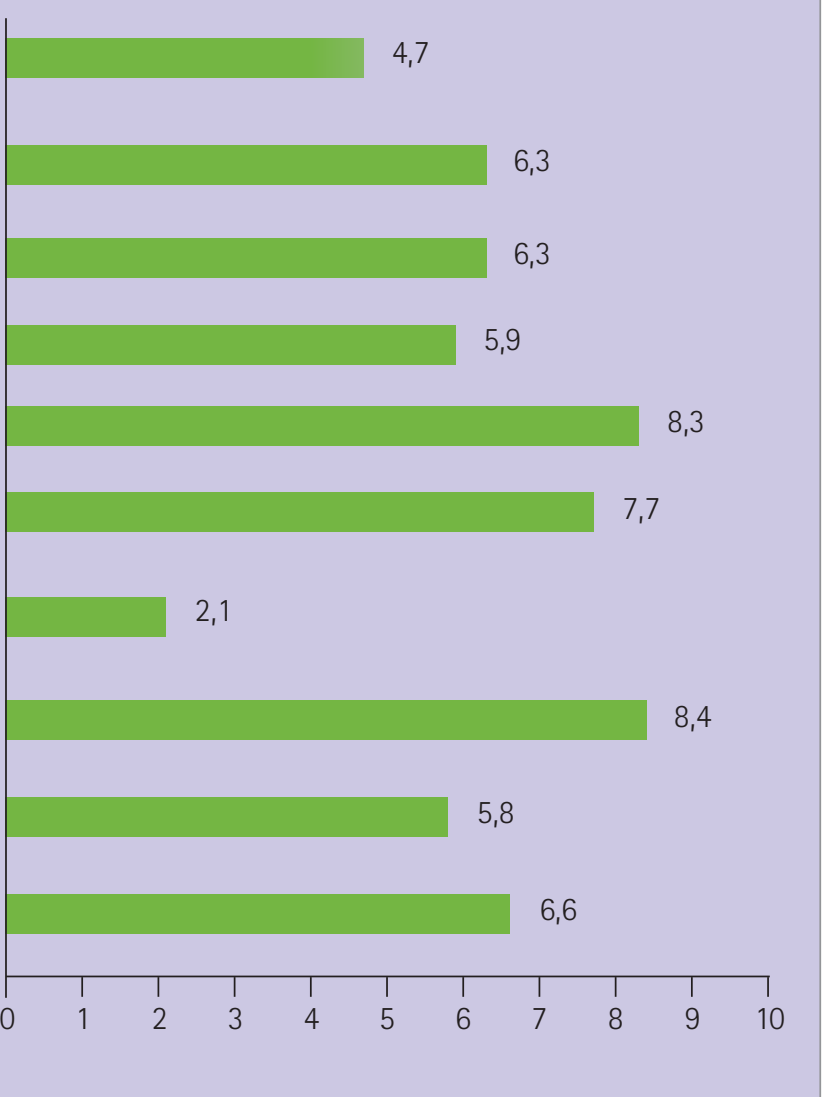

Figura 2. Valores medios de las respuestas de los farmacéuticos participantes a la encuesta de valoración

\section{Conclusiones}

SEFAC pone a disposición de los farmacéuticos comunitarios un $\mathrm{Li}$ bro de Incidencias cuya llevanza se ha mostrado útil en la mejora de la calidad asistencial en la farmacia comunitaria.

Los farmacéuticos participantes perciben que el registro de las incidencias en un Libro de incidencias les es de gran ayuda para la justificación de su actuación profesional en situaciones complejas o comprometidas.

Conforme a la definición dada de Incidencia para este estudio, se ha registrado una apreciable diversidad de ellas, que el farmacéutico comunitario ha resuelto.

La frecuencia de registros, la facilidad de manejo y la valoración de las farmacias participantes justificaría la difusión del Libro de Incidencias.

\section{Referencias bibliográficas}

1. Abellán, F [Coordinador]. Recetas sencillas para consultas complejas. Cómo resolver los 15 conflictos tipo en la relación asistencial farmacéutico-paciente. Madrid: SEFAC; 2009.

2. Abellán, F [Coordinador]. Manual de Consultas Bioéticolegales en la Farmacia Comunitaria. Madrid: SEFAC; 2012.

3. Abellán F. Nota informativa de la Comisión de Bioética de SEFAC [Editorial]. Farmacéuticos Comunitarios. 2011;3(1):5. 